\title{
RELACION DE LA MADUREZ VOCACIONAL CON EL RENDIMIENTO ACADEMICO, LA EDAD Y EL SEXO
}

\author{
RELATIONSHIPS BETWEEN CAREER MATURITY, AGE, SEX, \\ AND ACADEMIC ACHIEVEMENT
}

\author{
Daniel Anaya* \\ UNED
}

\section{RESUMEN}

El trabajo estudia las relaciones de la edad, el sexo y el rendimiento académico con las medidas de madurez vocacional o de la carrera proporcionadas por el Career Development Inventory - School Form. Los hallazgos, basados sobre una amplia muestra de estudiantes españoles de educación secundaria representativa a nivel nacional, constituyen una importante aportación a la validez del instrumento para su utilización en el colectivo estudiado.

Palabras clave: Madurez vocacional o de la carrera, evaluación, edad, sexo, rendimiento académico.

\begin{abstract}
This work studies relationships of age, sex, and academic achievement with career maturity measurements provided by Career Devepoment Inventory - School Form. The finds, based over an extensive sample of spanish high school students representative at national level, constitute an important contribution to instrument validity for its utilization with this collective.
\end{abstract}

Key words: Career maturity, assessment, age, sex, academic achievement.

Daniel Anaya es profesor de Diagnóstico en Educación en la Universidad Nacional de Educación a Distancia. Sus líneas de investigación, actualmente, están centradas en la elaboración y validación de recursos para el diagnóstico y la intervención educativa.

* Daniel Anaya es profesor de Diagnóstico en Educación en la Universidad Nacional de Educación a Distancia. Sus líneas de investigación, actualmente, están centradas en la elaboración y validación de recursos para el diagnóstico y la intervención educativa. 


\section{Introducción}

El concepto de madurez vocacional fue introducido por Super en 1955, definiéndolo como "el lugar alcanzado en el continuo del desarrollo vocacional desde la exploración al declive" (Super, 1955, p. 153). Con posterioridad, esta definición ha ido precisándose y, en concreto, Super y sus colaboradores la han entendido como la disposición o preparación para afrontar las tareas relacionadas con el desarrollo de la carrera que son apropiadas a cada etapa de la vida de la persona, lo que incluye la elaboración de las decisiones sobre la carrera que tienen que ver con el progreso a lo largo de la escolaridad, dentro del mundo del trabajo, y a lo largo de la etapa adulta hasta la madurez tardía y el retiro (Super y Overstreet, 1960; Jordaan y Heyde, 1979).

En el contexto de las teorías del desarrollo entonces disponibles, Super concibió las carreras como conformadas por una serie de etapas evolutivas caracterizada, cada una de ellas, por ciertas tareas apropiadas. La especificación de estas tareas correspondientes a cada etapa dio a Super la oportunidad de medir el progreso en dichas tareas mediante la comparación ipsativa de las ejecuciones del sujeto en distintos momentos de su vida o a través de la comparación con otros individuos que se encuentran en la misma etapa evolutiva y que, por consiguiente, contienden, supuestamente, con las mismas tareas.

Después de especificar las etapas del desarrollo de la carrera desde la temprana adolescencia hasta la edad de la jubilación o retiro, Super y sus asociados centraron sus esfuerzos en el estudio de la etapa de adolescencia y madurez temprana que, básicamente, se identificó con los estudiantes de secundaria y universitarios. Uno de los resultados de sus trabajos fue el Career Development Inventory (CDI), un instrumento dirigido a evaluar la madurez vocacional o de la carrera.

Originalmente, apareció en 1971 (Super, Bohn, Forrest, Jordaan, Lindeman y Thompson, 1971) y, al año siguiente, se publicó el Manual (Super y Forrest, 1972). Con posterioridad, surgieron el CDI-forma II y el CDI-forma III (Super, 1974), el primero dirigido a adultos $\mathrm{y}$, el segundo, con el fin de mejorar el CDI primitivo para aprehender mejor los aspectos cognitivos de la madurez vocacional.

Por último, en la primavera de 1979 apareció la más reciente versión del CDI (Super, Thompson, Lindeman, Jordaan y Myers, 1979). Dos años más tarde se publicó el Manual del Usuario (Thompson y Lindeman, 1981), que está dirigido a facilitar la investigación y el uso del instrumento, y en 1984 apareció el Manual Técnico (Thompson y Lindeman, 1984), que aporta abundante información acerca de las propiedades métricas de la prueba.

Esta última versión del CDI presenta dos formas: La School Form, dirigida a estudiantes de los grados $8^{\circ}$ al $12^{\circ}$-tramo equivalente a la etapa de educación secundaria de nuestro sistema educativo, desde $2^{\circ}$ de ESO a $2^{\circ}$ de Bachillerato-, aunque una de las escalas, Conocimiento del Grupo Ocupacional Preferido (PO), va destinada únicamente a alumnos de los grados $11^{\circ}$ y $12^{\circ}$-equivalentes a nuestros dos cursos de Bachillerato-, y la College and University Form, dirigida a estudiantes universitarios.

La Forma Escolar del CDI (CDI-School Form) ha sido objeto, por nuestra parte, de adaptación a la población española (Anaya y Repetto, 1992; Anaya y Repetto, 1998), con la exclusión de la escala de Conocimiento del Grupo Ocupacional Preferido (PO), que se dejó fuera debido a su no participación en la puntuación total y a su aplicabilidad restringida a sólo una parte del colectivo de interés. 


\section{Objetivo del estudio}

El presente estudio tiene como objeto descubrir las relaciones de la edad, el sexo y el rendimiento académico con las medidas de madurez vocacional proporcionadas por la Forma Escolar del CDI, con la finalidad de incrementar su validez en población española.

Actualmente, la validez, en relación a un instrumento, se entiende como el grado en el que todas las evidencias acumuladas respaldan las interpretaciones o inferencias pretendidas a partir de los datos proporcionados por el instrumento (American Educational Research Association, American Psychological Association, y National Council on Measurement in Education, 1999). Aunque estas evidencias pueden ser de muy diversa índole y estar referidas a cualquier dato empírico relacionado con aquél, son especialmente relevantes las relativas a aquellas características predichas para el instrumento por la teoría que enmarca el constructo medido.

En relación con el CDI, sus autores pronosticaron, entre otros hechos, que las dimensiones no cognitivas hipotetizadas estarían incorrelacionadas o escasamente relacionadas con medidas cognitivas como, por ejemplo, el rendimiento académico; que las puntuaciones en todas las dimensiones hipotetizadas incrementarían, en la mayoría de los casos, con la edad y el nivel académico, y que estas puntuaciones serían semejantes en ambos sexos. La comprobación de esas predicciones en relación con la versión española del CDI constituye, como antes se ha indicado, el objeto de este estudio. [Evidencias relativas a la estabilidad del constructo, a la estructura factorial y a la sensibilidad del instrumento para detectar cambios tras los tratamientos, entre otras, ya han sido previamente aportadas (Anaya y Repetto, 1992, 1998)].

\section{El inventario del desarrollo de la carrera - Forma escolar (CDI-SF)}

El CDI-SF está dirigido a facilitar una evaluación de la madurez vocacional en estudiantes de educación secundaria. En su edición original consta de cinco escalas primarias, pero en la versión española realizada por el autor se prescindió de la escala PO por las razones antes apuntadas. Esta versión, en consecuencia, está formada por las siguientes cuatro escalas básicas:

- Planificación de la Carrera (CP). Comprende 20 ítems de elección entre cinco alternativas de los que los 12 primeros están referidos al grado en el que el estudiante ha estado, hasta ahora, comprometido en actividades de planificación de la carrera, y los 8 restantes piden información sobre la cantidad de conocimiento del sujeto en relación con sus ocupaciones preferidas.

- Exploración de la Carrera (CE). Conformada por 20 ítems de elección entre cuatro alternativas, referidos los 10 primeros al grado en el que el estudiante acudiría a otras tantas fuentes de información o ayuda para realizar sus planes futuros de estudio y de trabajo, y los 10 restantes, al grado en el que esas mismas fuentes han dado ya al sujeto información útil para hacer sus planes futuros.

- Toma de Decisiones (DM). Está formada por 20 ítems consistentes en el planteamiento de otros tantos problemas de toma de decisiones acerca de la carrera y sobre los que el estudiante debe elegir, en cada caso, la mejor opción entre cuatro alternativas dadas. 
- Información sobre el Mundo del Trabajo (WW). Comprende 20 ítems de elección entre cuatro alternativas de los que los 10 primeros evalúan conocimiento acerca de las tareas adecuadas, según la teoría de Super, a las etapas de Exploración y Establecimiento Temprano, y los 10 restantes evalúan conocimiento de las ocupaciones.

Cada una de estas escalas básicas proporciona una puntuación independiente. Además, el CDI provee de una puntuación respecto de cada una de las siguientes escalas combinadas:

- Actitudes del Desarrollo de la Carrera (CDA), que surge de la combinación de las escalas CP y CE.

- Conocimiento y Habilidades del Desarrollo de la Carrera (CDK), que surge de la combinación de las escalas DM y WW.

- Total de Orientación de la Carrera (COT), que surge de la combinación de las cuatro escalas básicas, esto es, CP, CE, DM y WW. COT es una medida compuesta de los cuatro aspectos o dimensiones importantes de la madurez de la carrera y, según los autores, puede ser considerada como una medida aproximada de la madurez vocacional o de la carrera.

Los valores de estabilidad obtenidos con la versión española del CDI-SF, en intervalo de 6 meses, fluctúan entre .50 en la escala de Toma de Decisiones (DM) y .69 en la escala de Planificación de la Carrera (CP), siendo de .68 el correspondiente a la puntuación total de la escala (COT) (Anaya y Repetto, 1998), valores que son semejantes a los obtenidos con el CDI original utilizando un intervalo temporal similar (Hansen, 1985; Johnson, 1985; Thompson y Lindeman, 1984).

Factorialmente, el constructo medido se estructura en dos dimensiones: Una dimensión actitudinal o conativa, definida por la disposición del sujeto hacia tareas de planificación y exploración de la carrera (escalas CP y CE), y otra dimensión cognitiva, caracterizada por la habilidad para tomar decisiones sobre la carrera y para adquirir información sobre el mundo del trabajo (escalas DM y WW) (Anaya y Repetto, 1998). Esta estructura factorial sigue los resultados obtenidos en los estudios norteamericanos informados en el Manual del Usuario y en el Manual Técnico, así como los referidos en las revisiones efectuadas por Hansen (1985), Pinkney (1985) y Johnson (1985). Las hipotetizadas, por la teoría, dos dimensiones de la madurez vocacional (actitudinal y cognitiva) quedan, por consiguiente, bien respaldadas por la evidencia empírica.

\section{Descripción de la muestra}

El presente estudio está basado en una muestra de 2534 estudiantes de los cursos $3^{\circ}$ y $4^{\circ}$ de Educación Secundaria Obligatoria y $1^{\circ}$ y $2^{\circ}$ de Bachillerato representativa a nivel nacional. En la tabla 1 se presenta la distribución de la muestra por nivel académico y sexo.

\section{Presentación y discusión de resultados}

\section{Madurez vocacional y edad}

En sintonía con la mayoría de los estudios que han tratado esta cuestión, la variable edad se ha manejado, también aquí, en términos de nivel académico. La madurez vocacio- 
TABLA 1: Distribución de la muestra por nivel académico y sexo.

\begin{tabular}{|l|c|c|c|c|c|}
\hline \multirow{2}{*}{$\begin{array}{c}\text { Nivel } \\
\text { académico }\end{array}$} & \multicolumn{2}{|c|}{ Mujer } & Varón & & Total \\
\cline { 2 - 6 } & $\mathbf{n}$ & $\mathbf{\%}$ & $\mathbf{n}$ & $\mathbf{\%}$ & $\mathbf{n}$ \\
\hline $3^{\mathbf{0}} \mathrm{ESO}$ & 385 & 50.45 & 378 & 49.55 & 763 \\
\hline $4^{\circ} \mathrm{ESO}$ & 361 & 50.48 & 354 & 49.52 & 715 \\
\hline $1^{\circ} \mathrm{BACH}$ & 294 & 51.21 & 280 & 48,79 & 574 \\
\hline $2^{\circ} \mathrm{BACH}$ & 253 & 52.48 & 229 & 47.52 & 482 \\
\hline TOTAL & $\mathbf{1 2 9 3}$ & $\mathbf{5 1 . 5 3}$ & $\mathbf{1 2 4 1}$ & $\mathbf{4 8 . 4 7}$ & $\mathbf{2 5 3 4}$ \\
\hline
\end{tabular}

nal es un concepto evolutivo, que forma parte del desarrollo general del individuo, y se considera que los estudiantes de un mismo nivel académico se encuentran en un similar momento evolutivo o etapa del desarrollo.

En la tabla 2 aparece la puntuación media obtenida en cada escala por los estudiantes de cada uno de los niveles académicos de educación secundaria, así como el nivel de significación de la diferencia de medias entre cada par de grados académicos, que se ha contrastado mediante el estadístico $t$. [Las puntuaciones que aparecen en la tabla son puntuaciones típicas con media de 100 y desviación típica de 20. Estas puntuaciones se han empleado con objeto de facilitar la comparación entre las escalas y entre los grupos].

De los 42 contrastes de diferencias de medias que se muestran en la tabla 2, 38 han dado como resultado una diferencia altamente significativa, asociada a una probabilidad inferior a .001, 1 indica una diferencia asociada a una probabilidad inferior a .01, y sólo 3 no han resultado estadísticamente significativos.

Todas las escalas muestran un progresivo incremento a lo largo de la secundaria que resulta, en todos los casos, estadísticamente significativo al pasar de un nivel académico al si-

TABLA 2: Diferencias de medias entre niveles académicos.

\begin{tabular}{|c|c|c|c|c|c|c|c|c|c|c|}
\hline Escala & $\mathbf{3}^{\mathbf{0}} \mathbf{E}$ & $\mathbf{d}_{\mathbf{3}, \mathbf{4}}$ & $\mathbf{4}^{\mathbf{0}} \mathbf{E}$ & $\mathbf{d}_{\mathbf{4}, \mathbf{1}}$ & $\mathbf{1}^{\mathbf{0}} \mathbf{B}$ & $\mathbf{d}_{\mathbf{1}, \mathbf{2}}$ & $\mathbf{2}^{\mathbf{o}} \mathbf{B}$ & $\mathbf{d}_{\mathbf{3}, \mathbf{1}}$ & $\mathbf{d}_{\mathbf{3}, \mathbf{2}}$ & $\mathbf{d}_{\mathbf{4 , 2}}$ \\
\hline $\mathrm{CP}$ & 91.8 & $* *$ & 98.7 & $*$ & 102.1 & $* *$ & 112.4 & $* *$ & $* *$ & $* *$ \\
\hline $\mathrm{CE}$ & 91.9 & $* *$ & 98.1 & $* *$ & 102.4 & $* *$ & 112.7 & $* *$ & $* *$ & $* *$ \\
\hline $\mathrm{DM}$ & 93.1 & $* *$ & 97.8 & $* *$ & 105.9 & - & 107.1 & $* *$ & $* *$ & $* *$ \\
\hline $\mathrm{WW}$ & 94.0 & $* *$ & 99.1 & $* *$ & 104.5 & - & 104.6 & $* *$ & $* *$ & $* *$ \\
\hline $\mathrm{CDA}$ & 91.8 & $* *$ & 98.0 & $* *$ & 102.2 & $* *$ & 113.3 & $* *$ & $* *$ & $* *$ \\
\hline $\mathrm{CDK}$ & 93.5 & $* *$ & 98.5 & $* *$ & 104.6 & - & 107.0 & $* *$ & $* *$ & $* *$ \\
\hline $\mathrm{COT}$ & 92.9 & $* *$ & 98.2 & $* *$ & 103.4 & $* *$ & 109.8 & $* *$ & $* *$ & $* *$ \\
\hline
\end{tabular}

** prob. $<.001 ; \quad *$ prob. $<.01 ; \quad-$ prob. $>.01$

$\mathrm{d}_{3,4}=$ dif. entre $3^{\circ}$ y $4^{\circ}$ de ESO

$\mathrm{d}_{4,1}=$ dif entre $4^{\circ}$ de ESO y $1^{\circ}$ de BACH

$\mathrm{d}_{1,2}=$ dif entre $1^{\circ}$ y $2^{\circ}$ de BACH

$\mathrm{d}_{3,1}=$ dif entre $3^{\circ}$ de ESO y $1^{\circ}$ de BACH

$\mathrm{d}_{3,2}=$ dif entre $3^{\circ}$ de ESO y $2^{\circ}$ de BACH

$\mathrm{d}_{4,2}=$ dif entre $4^{\circ}$ de ESO y $2^{\circ}$ de BACH 
guiente, excepto en las escalas DM, WW Y CDK entre los cursos de $1^{\circ}$ y $2^{\circ}$ de Bachillerato. Estos datos están en línea con los aportados por otros trabajos (Dupont y Gingras, 1991; Patton, 2001; Post-Kammer, 1987; Thompson y Lindeman, 1981; Wallace-Broscious, Serafica y Osipow, 1994) que se han centrado, igualmente, en estos años de educación secundaria. Incluso, en lo que respecta a la diferencia no significativa en las escalas cognitivas entre los últimos cursos de esta etapa.

Las predicciones de la teoría respecto al incremento de la madurez vocacional en el transcurso de la edad/niveles académicos (Super, 1957, 1974; Super y Overstreet, 1960; Jordaan y Heyde, 1979) suponen que un instrumento dirigido a medir el constructo sea sensible a estos cambios. Nuestros hallazgos evidencian que, en el caso del CDI-SF, esto es así.

Por otra parte, desde un análisis más detallado de los datos, se observa que el incremento es, en términos generales, más fuerte en las escalas $\mathrm{CP}, \mathrm{CE}$ y CDA que en las escalas DM, WW y CDK. El componente actitudinal parte en $3^{\circ}$ de ESO desde un nivel de desarrollo inferior al componente cognitivo y alcanza un máximo en $2^{\circ}$ de Bachillerato superior al componente cognitivo. En el transcurso de los cuatro años, la escala CDA, por ejemplo, tiene un recorrido de 21.5 puntos frente a los 13.5 puntos logrado por la escala CDK. El componente cognitivo de la madurez vocacional, expresado en términos de habilidades para tomar decisiones y para adquirir información sobre el mundo del trabajo parece, por consiguiente, que se desarrolla más temprano y prácticamente, se estabiliza a partir de $1^{\circ}$ de Bachillerato. El componente actitudinal, concretado en las tareas de planificación y de exploración de la carrera, tiene un desarrollo más tardío, pero logra incrementos superiores hasta superar en $2^{\circ}$ de Bachillerato al componente cognitivo.

Otro dato interesante lo constituye la desigual secuencia de desarrollo seguida por las distintas dimensiones de la madurez vocacional. Las escalas actitudinales ofrecen el mayor salto cuantitativo entre $1^{\circ}$ y $2^{\circ}$ de Bachillerato, en tanto que en las escalas cognitivas, especialmente la escala DM, esta mayor progresión se observa entre $4^{\circ}$ de ESO y $1^{\circ}$ de Bachillerato. Es posible que el importante punto de decisión, en términos de la teoría de Super, que los estudiantes tienen al final del $2^{\circ}$ curso de Bachillerato esté detrás del fuerte aumento en estos alumnos de su implicación en tareas de planificación y de exploración de la carrera. Por otra parte, el notable incremento en los componentes cognitivos, especialmente en habilidades para tomar decisiones, que se produce entre $4^{\circ}$ de ESO y $1^{\circ}$ de Bachillerato tal vez tiene que ver con el mejor rendimiento académico que, como grupo, tienen los alumnos que, a partir de $4^{\circ}$ de ESO, deciden continuar sus estudios secundarios, pues, como se verá más adelante, son muchas las evidencias que apoyan la relación entre la dimensión cognitiva de la madurez vocacional y el rendimiento académico.

\section{Madurez vocacional y sexo}

La tabla 3 muestra la puntuación media obtenida en cada escala por las chicas y por los chicos de cada uno de los niveles académicos, así como el nivel de significación de las diferencias de esas medias dentro de un mismo nivel académico, que se ha contrastado mediante el estadístico $t$. [Las puntuaciones que aparecen en la tabla son puntuaciones típicas con media de 100 y desviación típica de 20. Estas puntuaciones se han empleado con objeto de facilitar la comparación entre las escalas y entre los grupos]. 
TABLA 3: Diferencias de medias entre chicas y chicos por nivel académico.

\begin{tabular}{|c|c|c|c|c|c|}
\hline \multirow{2}{*}{ Escala } & \multicolumn{2}{|c|}{$\mathbf{3}^{\mathbf{0}}$ ESO } & \multicolumn{2}{|c|}{$\mathbf{4}^{\mathbf{0}}$ ESO } & \multicolumn{2}{c|}{$\mathbf{1}^{\mathbf{0}}$ BACH } & \multicolumn{2}{c|}{$\mathbf{2}^{\mathbf{0}}$ BACH } \\
\cline { 2 - 6 } & $\mathbf{M} \mathbf{d} \mathbf{V}$ & $\mathbf{M} \mathbf{d} \mathbf{V}$ & $\mathbf{M} \mathbf{d} \mathbf{V}$ & $\mathbf{M} \mathbf{d} \mathbf{V}$ \\
\hline $\mathrm{CP}$ & $91.7-91.9$ & $98.2-99.1$ & $102.2-101.8$ & $112.0-113.1$ \\
\hline $\mathrm{CE}$ & $92.1-91.8$ & $97.7-98.5$ & $102.6-102.1$ & $111.9-113.1$ \\
\hline $\mathrm{DM}$ & $95.6 * * 90.4$ & $100.2 * * * 95.3$ & $107.4-104.3$ & $107.7-106.4$ \\
\hline $\mathrm{WW}$ & $96.4 * 91.7$ & $101.4 * 96.7$ & $105.9-102.9$ & $103.1-106.0$ \\
\hline $\mathrm{CDA}$ & $91.9-91.7$ & $97.5-98.7$ & $102.4-101.9$ & $112.1-114.2$ \\
\hline $\mathrm{CDK}$ & $95.8 * 91.1$ & $100.8 * 96.1$ & $106.7 * 102.3$ & $105.4-108.3$ \\
\hline $\mathrm{COT}$ & $94.3 * 91.4$ & $98.9-97.3$ & $104.6-102.0$ & $109.2-110.0$ \\
\hline
\end{tabular}

** prob. $<.001 ; \quad *$ prob. $<.01 ; \quad-$ prob. $>.01$

$\mathrm{M}=$ Media en mujeres

$\mathrm{V}=$ Media en varones

$\mathrm{d}=$ Diferencia de medias entre mujeres y varones

Como se puede apreciar, la tabla muestra pocos casos en los que las diferencias sexuales dentro de un mismo nivel académico son significativas; se circunscriben a las escalas cognitivas en los cursos $3^{\circ}$ y $4^{\circ}$ de ESO. Estos datos están en sintonía con otros estudios que han encontrado, igualmente, escasas diferencias sexuales en estos años de educación secundaria (Dupont y Gingras, 1991; Kelly y Colangelo, 1990; Thompson y Lindeman, 1984; Touma, 1998; Watson, Stead y De Jager, 1995). Además, cuando se han encontrado diferencias significativas ha sido, generalmente, en las escalas cognitivas y a favor de las chicas (Lokan, 1984; Luzzo, 1995; Petrone, 2000; Thompson y Lindeman, 1984; Westbrook, Cutts, Madison y Arcia, 1980), como ha ocurrido, también, en esta ocasión.

La teoría del desarrollo de la carrera que sirve de base al CDI predice mínimas diferencias sexuales en madurez vocacional. Es esperable, en consecuencia, que el CDI, cuyos ítems están deliberadamente expresados en términos unisexuales y que no fueron seleccionados para marcar las diferencias entre mujeres y hombres, provea de puntuaciones sexualmente indiferenciadas. Los resultados que muestra la tabla 3 son congruentes con lo pronosticado por la teoría, dado que de 28 contrastes realizados sólo en 7 las diferencias han sido estadísticamente significativas. Estas se han centrado en las escalas cognitivas, como se ha visto anteriormente, y, en especial, las diferencias alcanzan una mayor magnitud en la escala de Toma de Decisiones (DM).

La mayor puntuación obtenida por las chicas de los cursos $3^{\circ}$ y $4^{\circ}$ de ESO en las escalas cognitivas y, especialmente, en la escala de Toma de Decisiones (DM) parece estar ligada al mejor rendimiento académico que, en estos cursos, las chicas obtienen frente a sus compañeros varones -en Bachillerato desaparecen estas diferencias de rendimiento-. Son muchas las evidencias (Dupont y Dingras, 1991; Kelly y Colangelo, 1990; Lokan, 1984; Luzzo, 1995; Thompson y Lindeman, 1982, 1984; Watson et al., 1995; Westbrook et al., 1980), incluidas las aportadas en este estudio, como se verá en el siguiente apartado, que muestran que las escalas cognitivas están más altamente correlacionadas con medidas de rendimiento 
académico que las escalas actitudinales. Nuestros hallazgos, en línea con lo encontrado en otros trabajos -ver las anteriores referencias-, apoyan que existe una tendencia a que, en estos cursos, las chicas obtienen en las escalas cognitivas puntuaciones superiores a sus compañeros varones y que tales diferencias son consistentes con diferencias sexuales en rendimiento académico.

\section{Madurez vocacional y rendimiento académico}

La tabla 4 muestra los coeficientes de correlación de Pearson entre las distintas escalas del CDI-SF y el rendimiento académico en cada uno de los cuatro cursos de educación secundaria estudiados. [Como medida del rendimiento académico se ha utilizado la media aritmética de las calificaciones, expresadas en una escala de 0 a 10 puntos, obtenidas por el estudiante, en junio, en las asignaturas cursadas].

La puntuación Total de Orientación de la Carrera (COT) que, según los constructores del CDI, puede ser tomada como una medida de la madurez vocacional o de la carrera, aparece significativamente relacionada con el rendimiento académico en todos los cursos de secundaria. De acuerdo con esta información, la madurez vocacional y el rendimiento académico correlacionan de forma significativa. Pero esta información necesita ser precisada, dado que COT es una puntuación global obtenida de la combinación de los componentes actitudinal y cognitivo medidos por el CDI.

Observando los resultados a nivel de las escalas combinadas de Actitudes (CDA) y de Conocimiento y Habilidades del Desarrollo de la Carrera (CDK) se obtiene que, en tanto la primera está incorrelacionada con el rendimiento académico, la segunda presenta una correlación significativa con aquél. Finalmente, de la observación de los resultados a nivel de escalas básicas, se extrae que, mientras la incorrelación de la dimensión actitudinal con el rendimiento es semejante en sus componentes de planificación $(\mathrm{CP})$ y de exploración $(\mathrm{CE})$ de la carrera, la significativa correlación de la dimensión cognitiva con el rendimiento es más fuerte, en todos los casos, respecto del componente de toma de decisiones (DM) que respecto del componente de información sobre el mundo del trabajo(WW).

TABLA 4: Correlaciones con el rendimiento académico.

\begin{tabular}{|c|c|c|c|c|}
\hline Escala & $\mathbf{3}^{\mathbf{0}} \mathbf{E S O}$ & $\mathbf{4}^{\mathbf{0}} \mathbf{E S O}$ & $\mathbf{1}^{\mathbf{}} \mathbf{B A C H}$ & $\mathbf{2}^{\mathbf{0}} \mathbf{B A C H}$ \\
\hline $\mathrm{CP}$ & -.04 & .06 & -.08 & .07 \\
\hline $\mathrm{CE}$ & .02 & .05 & .10 & -.03 \\
\hline $\mathrm{DM}$ & $.72^{* *}$ & $.68^{* *}$ & $.48^{* *}$ & $.41^{* *}$ \\
\hline $\mathrm{WW}$ & $.61^{* *}$ & $.55^{* *}$ & $.45^{* *}$ & $.39^{* *}$ \\
\hline $\mathrm{CDA}$ & -.03 & .05 & .04 & .05 \\
\hline $\mathrm{CDK}$ & $.66^{* *}$ & $.61^{* *}$ & $.46^{* *}$ & $.40^{* *}$ \\
\hline $\mathrm{COT}$ & $.37^{* *}$ & $.32^{* *}$ & $.28^{* *}$ & $.26^{* *}$ \\
\hline
\end{tabular}

$* *$ prob. $<.001 ; \quad *$ prob. $<.01$ 
El constructo madurez vocacional o de la carrera medido por el CDI-SF es claramente bidimensional (Anaya y Repetto, 1998) y los resultados arrojados por este estudio muestran que la dimensión actitudinal o conativa está incorrelacionada con el rendimiento académico en tanto que la dimensión cognitiva correlaciona significativamente con él y, de una manera aún más destacada, su componente de habilidades para la toma de decisiones. Estos resultados son consistentes con los informados en los estudios americanos sobre la versión original (Thompson y Lindeman, 1982, 1984) y en la revisión efectuada por Westbrook (1984) y constituyen una evidencia más a favor de la predicción realizada por los constructores del CDI, a partir de la teoría (Super, 1957, 1974; Super y Forrest, 1972; Super et al., 1979; Thompson y Lindeman, 1982, 1984), de que las escalas actitudinales (no cognitivas) estarían incorrelacionadas o escasamente relacionadas con el rendimiento académico. Existen, parece ser, habilidades de base comunes al rendimiento académico y a la dimensión cognitiva de la madurez vocacional, pero las disposiciones sobre las que se asienta la dimensión actitudinal o conativa son, por lo visto, de carácter bien distinto a las implicadas en el rendimiento académico.

Respecto de la relación entre las escalas cognitivas y el rendimiento académico, un hallazgo adicional facilitado en la tabla 4 tiene que ver con su progresiva disminución en el transcurso de la secundaria. A medida que se avanza en nivel académico, los coeficientes de correlación disminuyen sistemáticamente. A lo largo de la secundaria, las habilidades subyacentes al rendimiento académico parece ser que tienen cada vez menos en común con las implicadas en la toma de decisiones sobre la carrera y en la adquisición de información sobre el mundo del trabajo.

\section{Conclusiones}

Del siguiente estudio se obtienen las siguientes conclusiones principales:

1. Las puntuaciones en todas las escalas del Career Development Inventory - School Form (CDI-SF) aumentan sistemáticamente con la edad, expresada en términos de nivel académico, entre los cursos de $3^{\circ}$ de ESO y $2^{\circ}$ de Bachillerato. Estos resultados son consistentes con los encontrados en otros estudios con el CDI y con lo hipotetizado por la teoría a este respecto.

2. Las diferencias sexuales dentro de un mismo nivel académico son mínimas en las puntuaciones de las distintas escalas del CDI-SF. Sólo han resultado significativas, a favor de las chicas, las diferencias en las escalas cognitivas en $3^{\circ}$ y $4^{\circ}$ de ESO, que son consistentes con diferencias sexuales en rendimiento académico. Los hallazgos están en la línea de los informados en otros trabajos con el CDI y, en términos generales, apoyan la indiferenciación sexual del constructo previsto en la teoría.

3. Las escalas actitudinales del CDI-SF están incorrelacionadas con el rendimiento académico. Las escalas cognitivas, por el contrario, obtienen correlaciones significativas en todos los niveles académicos estudiados aunque, sistemáticamente, tienden a decrecer conforme aquéllos avanzan. Los datos están en sintonía con los aportados en otros estudios con el CDI y con lo hipotetizado por la teoría en este sentido. 
4. Estos hallazgos con el CDI-SF, obtenidos sobre una amplia muestra representativa a nivel nacional, constituyen una importante aportación a la validez del instrumento para su utilización con estudiantes españoles de educación secundaria.

\section{Referencias bibliográficas}

American Educational Research Association, American Psychological Association, \& National Council on Measurement in Education (1999). Standards for educational and psychological testing. Washington, DC: American Educational Research Association.

Anaya, D. \& Repetto, E. (1992). Adaptación española del Career Development Inventory - School Form. Un estudio piloto. No publicado. UNED.

Anaya, D. \& Repetto, E. (1998). "Estudio de la madurez vocacional de los estudiantes españoles de educación secundaria mediante el Career Development Inventory - School Form". Educación XXI, 1: 159-174.

Dupont, P. \& Gingras, M. (1991). "Des besoins d'orientation au secondaire: nécessité de nouvelles stratégies d'éducation à la carriére". Revue Canadienne de Counseling, 25 (2): 239-249.

Hansen, J. C. (1985). "Career Development Inventory". Measurement and Evaluation in Counseling and Development, 17: 220-224.

Johnson, S. D. (1985). "Career Development Inventory". En D. J. Keyse \& R. C. Sweetland (Eds.) Test critiques: Volume IV. Kansas City, MO: Test Corporation of America.

Jordaan, J. P. \& Heyde, M. B. (1979). Vocational maturity during the high-school years. Nueva York: Teachers College.

Kelly, K. R. \& Colangelo, N. (1990). "Effects of academic ability and gender on career development". Journal of the Education of the Gifted, 13: 168-175.

Lokan, J. (1984). Manual for the Career Development Inventory - Australian Edition. Melbourne: Australian Council for Educational Research.

Luzzo, D. A. (1995). "The relationship between career aspiration current occupation congruence and the career maturity of undergraduates". Journal of Employment Counseling, 32: 132-140.

Patton, W. (2001). "Developmental issues in career maturity and career decision status". Career Development Quarterly, 49 (4): 336-351.

Petrone, M. M. (2000). "Measuring competence for career decision making". Dissertation Abstract International: Section B: The Sciences \& Engineering, 61 (6-B): 3308.

Pinkney, J. W. (1985). "Review of Career Development Inventory". En J. V. Mitchell (Ed.) Ninth Mental Measurement Yearbook. Lincoln: University of Nebraska.

Post-Kammer, P. (1987). "Intrinsic and extrinsic work values and career maturity of $9^{\text {th }}$ and $11^{\text {th }}$ grade boys and girls". Journal of Counseling and Development, 65: 420-423.

Super, D. E. (1955). "Dimensions and measurement of vocational maturity". Teachers College Records, 57: 151-165.

Super, D. E. (1974). Measuring vocational maturity for counseling and evaluation. Washington, DC: National Vocational Guidance Association.

Super, D. E.; Bohn, M. J.; Forrest, D. J.; Jordaan, J. P., Lindeman, R. H. \& Thompson, A. S. (1971). Career Development Inventory. No publicado. Columbia University.

Super, D. E. \& Forrest, D. J. (1972). Career Development Inventory Form I: Preliminary Manual. Nueva York: Teachers College. 
Super, D. E. y Overstreet, P. L. (1960). The vocational maturity of ninth-grade boys. Nueva York: Teachers College.

Super, D. E., Thompson, A. S., Lindeman, R. H., Jordaan, J. P. \& Myers, R. A. (1979). Career Development Inventory. Palo Alto, CA: Consulting Psychologists Press.

Thompson, A. S. \& Lindeman, R. H. (1981). Career Development Inventory, Volume 1: Users' Manual. Palo Alto, CA: Consulting Psychologists Press.

Thompson, A. S. \& Lindeman, R. H. (1984). Career Development Inventory, Volume 2: Technical Manual. Palo Alto, CA: Consulting Psychologists Press.

Touma, S. G. (1998). "Career maturity and high school students: The effects of four variables on the career maturity of high school seniors". Dissertation Abstracts International Section A: Humanities and Social Sciences, 58 (7-A): 2551.

Wallace-Broscious, A., Serafica, F. C. \& Osipow, S. H. (1994). "Adolescent career development: Relationships to self-concept and identity status". Journal of Research on Adolescence, 4 (1): 127149.

Watson, M. B., Stead, G. B. \& De Jager, A. C. (1995). "The career development of black and white south African university students". International Journal for the Advancement of Counseling, 18: 39-47.

Westbrook, B. W. (1983). “Career maturity”. En W. B. Walsh \& S. H. Osipow (Eds.) Handbook of vocational psychology. Hillsdale, NJ: Erlbaum.

Westbrook, B. W., Cutts, C. C., Madison, S. S. \& Arcia, M. (1980). "The validity of the Crites model of career maturity". Journal of Vocational Behavior, 16: 249-281.

Fecha de recepción: 22-02-03

Fecha de revisión: 12-02-04

Fecha de aceptación: 12-05-04 